\section{EXTRA-ORAL SUCTION UNIT LAUNCHED}

The OPTIMA EOS 350 device is designed for dental practices to effectively capture droplets and airborne aerosols from treatments. As an extra-oral suction unit, the device will help to ensure patient comfort during usage.

The OPTIMA EOS 350 unit has a triple layer HEPA H14 filter, which traps 99.995\% of particles that are $\geq 0.3 \mu \mathrm{m}$. The double UVC lamps positioned before the HEPA filter inactivate virus and bacteria that are trapped by the filter. Through its Intelligent Airflow Dynamics, the exhaust air is released from a rear-mounted air vent at the top of the system to prevent blow-up of any dust particles or contaminants from floor surfaces. The air vent located at the rear enlarges comfort during treatment and circulates clean air in the room.

Other features of the OPTIMA EOS 350 extra-oral suction unit are:

- Intelligent control panel with digital indicator and adjustable power levels

- Easy to disinfect and with an auto HEPA filter replacement reminder

- Optimised height for easy handling and lightweight design for easy moving between surgeries using ergonomically placed pull handles

- Airtight casing - prevents contaminants

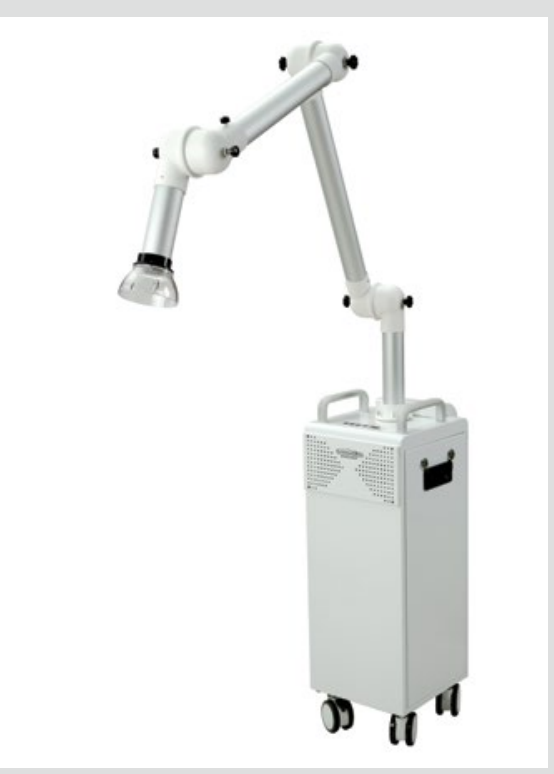

from escaping plus reduces noise

- Quiet operation at 58db (air-conditioning volume) and silent wheels with minimal noise.

The OPTIMA EOS 350 unit is available through Henry Schein Dental in the UK. For more information, visit: https:// www.hsdequipment.co.uk/product/ optima-eos-350.

\title{
A BEAUTIFUL AND SAFE DENTAL CHAIR
}

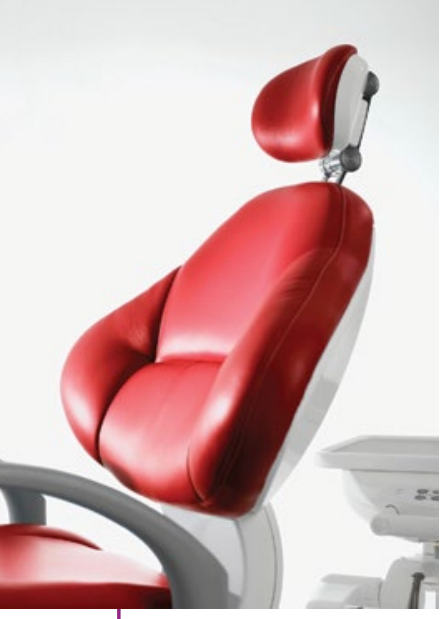

ensure the rigorous demands of a healthcare environment. The meticulous Japanese manufacturing process achieves both strength and longevity, making their fabrics naturally antimicrobial as well as ink and stain resistant.

Aesthetics must be matched with functionality, particularly in the current environment. Belmont has recently launched a range of care products which ensures the safety as well as the aesthetics of their equipment. There are two products in the chair maintenance range - B100 \& B300. Chairs need to be wiped down between patients and for such regular use you need a fast-acting upholstery cleaner that will not damage artificial leather; B300 does just that. It has reduced levels of alcohol so that over time it will prevent brittle, cracked surfaces. More importantly, it fulfils European virucidal standards and is effective against all enveloped viruses as well as the new type of coronavirus (SARS-CoV-2).

The perfect adjunct to this is B100, an intensive cleaner designed for occasional use, to remove stains of all kinds as well as discoloration. Despite its rigorous cleaning action, it will not damage your upholstery. In conjunction with B300, it will keep your chair looking beautiful as well as safe in the new environment in which practices now find themselves working.
AN EVENT THE WHOLE TEAM WON'T WANT TO MISS

The ADI Team Congress 2021 will deliver learning and networking opportunities that you and your team won't want to miss.

To be held on 6-8 May 2021 at

Manchester Central, the event is entitled 'The Great Debate - Current Dilemmas in Dental Implantology' The programme will focus on emerging treatment concepts and techniques, and internationally renowned clinicians will come together to share their insights and evaluations of different solutions.

There will also be moderated discussions and debates to further explore the clinical- and evidence-based approaches highlighted. Delegates will be encouraged to join the conversations which are sure to continue long after the Congress ends.

With sessions for all members of the team, it really is an all-inclusive event.

ADI members can attend the ADI Team Congress for discounted rates. Join today.

\section{LATEX-FREE PROTECTION \\ Did you know that professionals who routinely wear latex gloves are more likely to develop latex allergies in the future? \\ To combat this, Initial Medical has a huge array of gloves available for professionals, meaning that you need never worry about latex allergies again. Available in a wide selection of sizes and colours, the glove ranges from Initial Medical also include an expansive choice of materials, meaning that you can always find the perfect pair for all of your staff. \\ Prevent allergies and protect your hands by contacting Initial Medical today. \\ For further information visit www. initial.co.uk/medical or call 0870850 4045.}

If you would like to promote your products or services direct to the dental industry in BDJ Team, call Andy May on 02078434785 or emaila.may@nature.com 